\title{
Convex modeling of energy buffers in power control applications
}

\author{
Nikolce Murgovski ${ }^{*}$ Lars Johannesson ${ }^{* * *}$ Jonas Sjöberg* \\ * Department of Signals and Systems, Charmers University of \\ Technology, 41296 Gothenburg, Sweden (e-mail: \\ nikolce.murgovski@chalmers.se). \\ ** Viktoria Institute, 41756 Gothenburg, Sweden.
}

\begin{abstract}
This paper describes modeling steps for presenting energy buffers as convex models in power control applications. Except obtaining the optimal control, the paper also shows how convex optimization can be used to simultaneously size the energy buffer while optimally controlling a trajectory following system. The energy buffers are capacitors and batteries with quadratic power losses, while the resulting convex problem is a semidefinite program. The convex modeling steps are described through a problem of optimal buffer sizing and control of a hybrid electric vehicle. The studied vehicle is a city bus driven along a perfectly known bus line. The paper also shows modeling steps for alternative convex models where power losses and power limits of the energy buffer are approximated. The approximated models show significant decrease in computation time without visible impact on the optimal result.
\end{abstract}

Keywords: hybrid electric vehicle, convex optimization, ultracapacitors and batteries, optimal dimensioning and control

\section{INTRODUCTION}

The environmental pollution legislations, the need for lowering dependency on petroleum and the improved driveline efficiency, are main reasons that hybrid electric vehicles (HEVs) are being increasingly developed by vehicle companies. HEV drivelines utilize an energy buffer, typically a battery and/or an ultracapacitor, and one or more electric machines (EMs) to supplement the output of the internal combustion engine (ICE). This additional degree of freedom in HEVs allows for a more efficient operation, due to: a possibility to recover braking energy by using the EMs as generators and storing the energy in the buffer; ability to shut down the ICE during idling and low load demands; possibility to run the ICE at more efficient load conditions while storing the excess energy in the buffer. See e.g. Guzzella and Sciarretta (2007) for a detailed overview of hybrid vehicles.

Perhaps the highest potential of HEV technology has been found in public transportation. The usage of electric city buses dates back to 1895 , but HEV city buses are being most seriously considered in public transportation in the last decade (Anderson and Anderson, 2010). This is because buses spend significant time in idling or low load demands where the ICE has poor efficiency. Therefore, an HEV city bus equipped with a carefully sized electric buffer may improve the powertrain efficiency at most of the low load demands along the bus line. The cost effectiveness of the vehicle, in this case, will be directly affected by the type and size of the energy buffer, i.e. its power rating and energy capacity.

However, obtaining the optimal buffer size is not trivial. This is because the optimal buffer size depends on the drive patterns and topography along the bus line, varying factors such as fuel and buffer prices, and how well adapted the energy management strategy is to the bus line (Johannesson et al., 2009). For HEV city buses the energy management strategy decides the operating points of the ICE and thereby when and at which rate the energy buffer is to be discharged. A badly tuned energy management may lead to a non-optimal size of the energy buffer (Moore, 1997). Hence, to overcome this problem, both the size of the energy buffer and the energy management need to be optimized simultaneously.

The problem of optimal sizing and control of HEVs is traditionally solved by Dynamic Programming (Bellman, 1957), for which vast number of scientific articles are available (Zoelch and Schroeder, 1998; Kim and Peng, 2007; Sundström et al., 2010; Moura et al., 2010; Murgovski et al., 2011). The main advantage with DP is the capability to use nonlinear, non-convex models of the components consisting of continuous and integer (mixed-integer) optimization variables. However, a serious limitation of DP is that the computation time increases exponentially with the number of state variables (Bellman, 1957). As a consequence, the powertrain model is typically limited to only one or possibly two continuous state variables. Moreover, since DP operates by recursively solving a smaller subproblem for each time step, the second limitation of DP is that it is not possible to directly include the component sizing into the optimization. Instead, DP must be run in several loops to obtain the optimal control over a grid of component sizes, increasing computation time even further.

In a more recent study by Murgovski et al. (2012), convex optimization has been used to simultaneously size a 


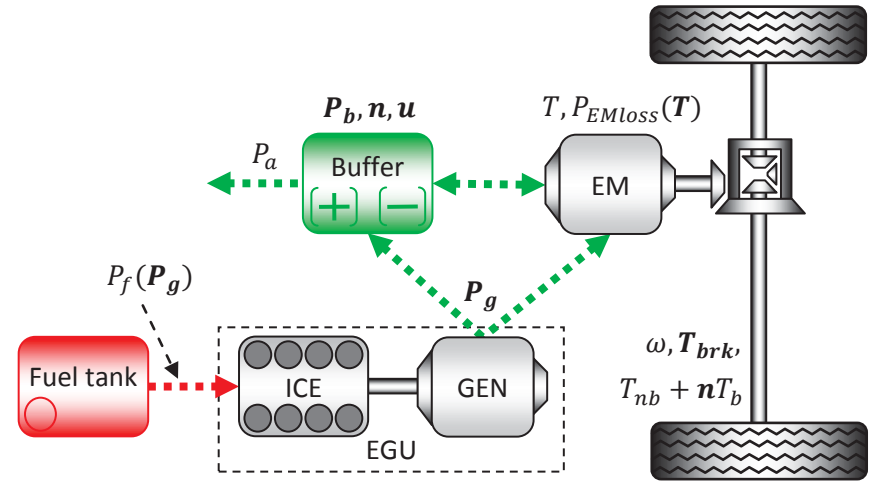

Fig. 1. Series PHEV powertrain model.

battery while optimally controlling $\mathrm{HEV}$ powertrains in series and parallel topology. The study considered approximated quadratic losses for the powertrain components and showed that the error due to the convexifying approximations is small as long as the battery open circuit voltage is nearly constant within the operating state of charge (SOC) interval. Due to this limitation the optimization strategy is suitable only for certain battery types and not for ultracapacitors.

This paper is an extension of the work of Murgovski et al. (2012) and shows modeling steps to allow for simultaneous sizing of ultracapacitors while optimally controlling HEVs via convex optimization techniques. Moreover, the proposed method also allows for sizing of batteries with nearly linear voltage-SOC dependency. Both the ultracapacitor and the battery are modeled with quadratic losses and the resulting convex problem is a semidefinite program (Boyd and Vandenberghe, 2004). The paper also shows modeling steps for alternative convex models where power losses and power limits of the energy buffer are approximated. By this, the computation time is decreased up to $50 \%$, i.e. the computation time of the battery sizing problem is decreased from about $15 \mathrm{~s}$ to about $7.5 \mathrm{~s}$ in average, without an optimization error.

The paper is outlined as follows: problem formulation and modeling details are described in Section 2; the convex modeling steps are discussed in Section 3; examples of capacitor and battery sizing are given in Section 4; and the paper is ended with conclusion in Section 5.

\section{PROBLEM FORMULATION}

This section gives a background on the bus line and the HEV powertrain model and formulates the energy buffer sizing problem.

\subsection{Bus line and powertrain model}

The studied HEV bus includes a powertrain in a series topology (Guzzella and Sciarretta, 2007), where there is no direct mechanical link between its combustion engine and the wheels (Fig. 1). Instead, the wheels are driven by an EM that receives energy from the electric buffer and/or an engine-generator unit (EGU).

The bus is driven on a bus line described by a road gradient and demanded velocity which are known at each point of
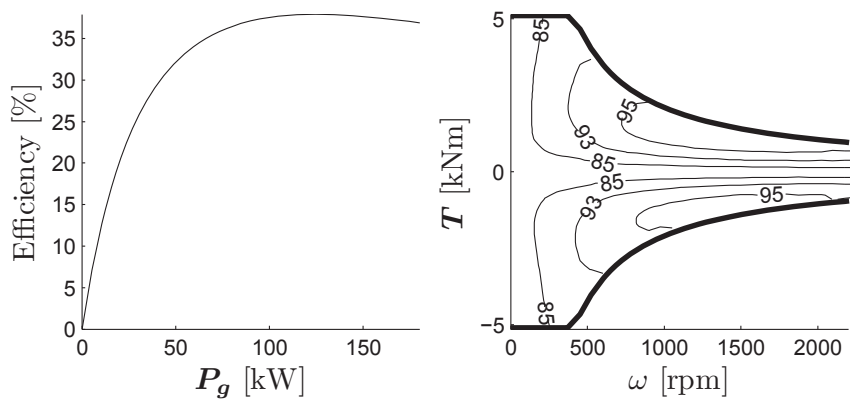

Fig. 2. Model of the EGU, left, and the EM, right. The thin lines represent efficiency and the thick lines are torque bounds of the EM.

time. The velocity and force demands from the bus line can be translated into an angular velocity $\omega(t)$ and torque $T_{n b}(t)+\boldsymbol{n} T_{b}(t)$ on the shaft between the EM and the differential. The two terms, $T_{n b}(t)$ and $\boldsymbol{n} T_{b}(t)$, represent the torque demanded from the powertrain without an energy buffer, and the torque from the increase in mass due to the $\boldsymbol{n}$ cells in the energy buffer, respectively. Both terms can be derived directly from the demanded acceleration and speed on the bus line and the known vehicle parameters, such as inertia, aerodynamic drag, rolling resistance, wheels radius, etc. See Guzzella and Sciarretta (2007) for details.

The EM, which delivers a torque $\boldsymbol{T}(t)$, is designed to be able to meet the high torque demands. Moreover, the EM is also used to recuperate braking energy up to the point when either its torque limit $T_{\min }(\omega(t))$, or the buffer charging limit is reached, after which friction brakes are used to handle the remaining braking torque $\boldsymbol{T}_{\boldsymbol{b} \boldsymbol{r} \boldsymbol{k}}(t)$, i.e.

$\boldsymbol{T}(t)=T_{n b}(t)+\boldsymbol{n} T_{b}(t)-\boldsymbol{T}_{\boldsymbol{b} \boldsymbol{r} \boldsymbol{k}}(t)$.

The optimization variables, e.g. $\boldsymbol{T}(t), \boldsymbol{n}, \boldsymbol{T}_{\boldsymbol{b} \boldsymbol{r} \boldsymbol{k}}(t)$, are marked in bold in order to improve readability.

The powertrain electric path is described by a power balance

$\boldsymbol{T}(t) \omega(t)+P_{E M l o s s}(\cdot)=\boldsymbol{P}_{\boldsymbol{b}}(t)+\boldsymbol{P}_{\boldsymbol{g}}(t)-P_{a}$

that relates the EM electric power, left side of the equality, to the energy buffer power $\boldsymbol{P}_{\boldsymbol{b}}(t)$, the EGU power $\boldsymbol{P}_{\boldsymbol{g}}(t)$ and the power consumed by auxiliary devices $P_{a}$. The losses of the EM, which also include the losses of the power electronics, are modeled as a quadratic function on $\boldsymbol{T}(t)$,

$P_{\text {EMloss }}(\cdot)=b_{0}(\omega(t)) \boldsymbol{T}^{2}(t)+b_{1}(\omega(t)) \boldsymbol{T}(t)+b_{2}(\omega(t))$ with speed dependent coefficients where $b_{j}(\omega(t)) \geq 0, j \in$ $\{0,2\}, \forall t \in\left[t_{0}, t_{f}\right]$. The initial and final time are denoted by $t_{0}$ and $t_{f}$, respectively, and the symbol - is used as a compact notation to indicate a function of optimization variables.

The EGU is also modeled with quadratic losses

$P_{f}\left(\boldsymbol{P}_{\boldsymbol{g}}(t), t\right)=a_{0} \boldsymbol{P}_{\boldsymbol{g}}^{\mathbf{2}}(t)+a_{1} \boldsymbol{P}_{\boldsymbol{g}}(t)+a_{2} e(t)$

with coefficients $a_{j} \geq 0, j \in\{0,2\}$, where $e(t)$ is a binary variable that is needed to allow for zero fuel power $P_{f}\left(\boldsymbol{P}_{\boldsymbol{g}}(t), t\right)$, i.e. to remove the idling losses $a_{2}$ when the engine is off. The EGU and the EM efficiencies are illustrated in Fig. 2.

The engine on/off control is decided prior to the optimization using heuristics that turn the engine on if the power 

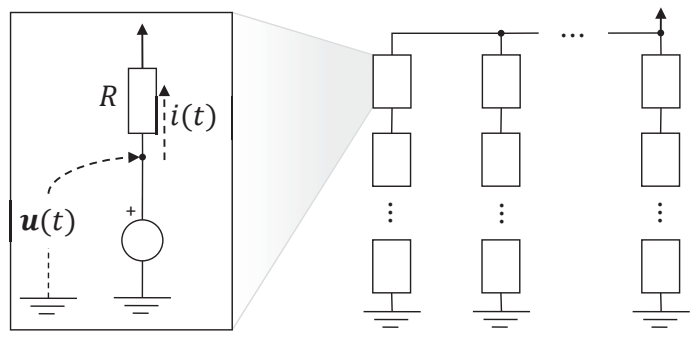

Fig. 3. Model of the energy buffer cell and pack. Cells are either ultracapacitors or batteries.

of the vehicle without an energy buffer exceeds a threshold $P_{o n}^{*}$, i.e.

$e(t)= \begin{cases}1, & T_{n b}(t) \omega(t)>P_{o n}^{*} \\ 0, & \text { otherwise }\end{cases}$

The optimal power threshold $P_{o n}^{*}$ is found by iteratively solving the optimization problem for several values of $P_{\text {on }}$ within the power range of the vehicle. The detailed procedure can be found in Murgovski et al. (2012), where it has been shown that these heuristics give small error to the global optimum. In this work $e(t)$ is considered predecided, aiming the focus on the convex modeling of the energy buffer.

\subsection{Energy buffer}

The energy buffer consists of $\boldsymbol{n}$ identical cells which could either be ultracapacitors or batteries. The cells are equally divided in parallel strings, with the strings consisting of cells connected in series, as in Fig. 3.

Ultracapacitor cell The model of the ultracapacitor cell can be described by the following equations

$$
\begin{aligned}
& i(\cdot)=\frac{1}{2 R}\left(\boldsymbol{u}(t)-\sqrt{\boldsymbol{u}^{2}(t)-\frac{4 R \boldsymbol{P}_{\boldsymbol{b}}(t)}{\boldsymbol{n}}}\right) \\
& i(\cdot) \in\left[i_{\text {min }}, i_{\text {max }}\right] \\
& \boldsymbol{P}_{\boldsymbol{b}}(t) \leq \frac{\boldsymbol{u}^{2}(t) \boldsymbol{n}}{4 R} \\
& \dot{\boldsymbol{u}}(t)=-\frac{1}{C} i(\cdot) \\
& \boldsymbol{u}(t) \in\left[s_{\min }, 1\right] u_{\max } \\
& \boldsymbol{u}\left(t_{f}\right)=\boldsymbol{u}\left(t_{0}\right)=u_{\max } s_{0}
\end{aligned}
$$

with $i(\cdot)$ and $\boldsymbol{u}(t)$ denoting the current and open circuit voltage of one cell. The cells are characterized by an inner resistance $R$, capacitance $C$, current limits $i_{\min }, i_{\max }$ and maximum voltage $u_{\max }$. The capacitor SOC, $s(t)=$ $\boldsymbol{u}(t) / u_{\max }$, is not allowed to drop below a limit $s_{\min }$ (typically greater than $30 \%$, or about $10 \%$ of the total energy content), as this energy is to be used for starting the engine, unplanned acceleration, or need for auxiliary power. The operation is charge sustaining by (6f) with an initial SOC $s_{0}$.

Note that (6a) has been derived from

$\boldsymbol{P}_{\boldsymbol{b}}(t)=\left(\boldsymbol{u}(t) i(\cdot)-R i^{2}(\cdot)\right) \boldsymbol{n}$

which shows that the power of each cell $\boldsymbol{P}_{\boldsymbol{b}}(t) / \boldsymbol{n}$ is equal and does not depend on the configuration of cells (series/parallel), but rather on the total number of cells. Therefore, in the rest of the paper the sizing problem will

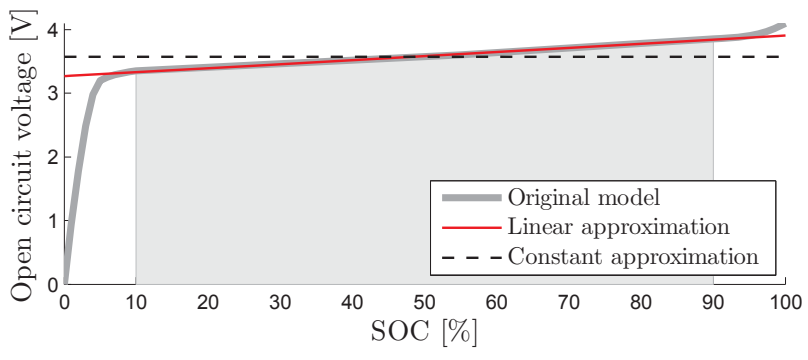

Fig. 4. Model of the battery cell open circuit voltage. The shaded region represents the allowed SOC range.

focus only on determining the total number of cells in the pack.

In the optimization $\boldsymbol{n}$ has a real value that indicates the total pack capacity. It can be expected that rounding this variable to the nearest integer gives small error if results point to large number of cells. This will generally be the case if the cells are chosen small.

Battery cell The model of the battery SOC is described and constrained by

$\dot{\boldsymbol{s}}(t)=-\frac{1}{Q} i(\cdot)$
$\boldsymbol{s}(t) \in\left[s_{\min }, s_{\text {max }}\right]$
$\boldsymbol{s}\left(t_{f}\right)=\boldsymbol{s}\left(t_{0}\right)=s_{0}$

where $Q$ is the cell capacity. The cell current and pack power are expressed exactly as in (6a)-(6c).

Instead of the cell voltage, the optimization variable describing cell dynamics is $\boldsymbol{s}(t)$, while the open circuit cell voltage is a nonlinear function of $s(t)$, as in Fig. 4. The usable $\mathrm{SOC}$ range is limited in order to extend the battery cycle and calendar life (Burke, 2007).

This work assumes constant cell resistance and capacitance for both the battery and the ultracapacitor. If this is not the case, the convex modeling steps presented in the rest of the paper cannot be applied directly.

\subsection{The non-convex optimization problem}

The studied optimization problem is formulated to minimize a sum of operational cost for consumed fuel on the bus line and cost for the energy buffer (9a), weighted by two coefficients $w_{f}$ [currency/kWh] and $w_{b}$ [currency] which transform the fuel power and the buffer size into a single unit. The optimization is subject to constraints invoked by the powertrain model, forming the nonlinear optimization problem

minimize

$w_{f} \int_{t_{0}}^{t_{f}} P_{f}\left(\boldsymbol{P}_{\boldsymbol{g}}(t), t\right) d t+w_{b} \boldsymbol{n}$

$\forall t \in\left[t_{0}, t_{f}\right]$, subject to

$\begin{cases}(6 \mathrm{~b})-(6 \mathrm{f}), & \text { for the ultracapacitor, or } \\ (6 \mathrm{~b}),(6 \mathrm{c}) \text { and }(8), & \text { for the battery, and }\end{cases}$

$T(t) \geq \max \left\{T(\omega(t)) T_{b}(t)+n T_{b}(t)\right\}$

$\boldsymbol{T}(t) \omega(t)+P_{\text {EMloss }}(\boldsymbol{T}(t), t) \leq \boldsymbol{P}_{\boldsymbol{b}}(t)+\boldsymbol{P}_{\boldsymbol{g}}(t)-P_{a}$

$\boldsymbol{P}_{\boldsymbol{g}}(t) \in\left[0, P_{\operatorname{gmax}} e(t)\right]$

$\boldsymbol{n} \geq 0$ 
with optimization variables $\boldsymbol{n}, \boldsymbol{P}_{\boldsymbol{g}}(t), \boldsymbol{P}_{\boldsymbol{b}}(t), \boldsymbol{T}(t) \forall t \in$ $\left[t_{0}, t_{f}\right]$ common for the two HEVs with different energy buffers. Additionally, the HEV with the ultracapacitor has $\boldsymbol{u}(t)$, while the HEV with the battery has $\boldsymbol{s}(t)$ as an optimization variable. The maximum EGU power is denoted by $P_{g \max }$.

The constraints (1) and (2) have been relaxed with inequalities in (9b) and (9c), respectively, and the braking torque has been taken out of the optimization problem. This does not change the optimal result, since at the optimum (9c) will hold with equality as otherwise energy will be wasted unnecessarily. Similarly, (9b) will also hold with equality, except during braking when the vehicle cannot recuperate all the braking energy. Then, the remaining energy is dissipated at the friction brakes and the optimal braking torque can be obtained directly from (1). See Murgovski et al. (2012) for details.

\section{CONVEX MODELING}

This section gives a brief background on convex optimization and remodels the energy buffer to give a convex optimization problem. The convex modeling steps follow a disciplined methodology, where the convexity of complex functions is verified using operations that preserve convexity of elementary convex functions (Boyd and Vandenberghe, 2004).

\subsection{Convex problem in a general form}

A convex problem can be written in the form

minimize $f_{0}(\boldsymbol{x})$

subject to $f_{j}(\boldsymbol{x}) \leq 0, \quad j=1, \ldots, m$

$h_{l}(\boldsymbol{x})=0, \quad l=1, \ldots, p$

$\boldsymbol{x} \in \mathcal{X}$

where $\mathcal{X} \subseteq \mathbb{R}^{n}$ is a convex set, $f_{j}(\boldsymbol{x}), j=0, \ldots, m$ are convex functions and $h_{l}(\boldsymbol{x})$ are affine functions in the optimization variables $\boldsymbol{x}$ (Boyd and Vandenberghe, 2004). The problem (9) is not convex due to the non-convex function $i(\cdot)$ and the state constraint $(6 \mathrm{~d})$, or $(8 \mathrm{a})$, that ties a nonlinear function with equality.

\subsection{Convex ultracapacitor model}

Modeling the ultracapacitor in a convex form is straight forward and requires only two steps, a variable change from cell voltage to pack energy, and relaxation with inequality.

Convexification steps By replacing the cell voltage with the pack energy

$\boldsymbol{E}(t)=\frac{C \boldsymbol{u}^{2}(t) \boldsymbol{n}}{2} \Rightarrow \dot{\boldsymbol{E}}(t)=C \boldsymbol{n u}(t) \dot{\boldsymbol{u}}(t)$

and by relaxing with inequality, (6d) can be replaced by

$\dot{\boldsymbol{E}}(t) \leq-\frac{1}{R C}\left(\boldsymbol{E}(t)-\sqrt{\boldsymbol{E}(t)\left(\boldsymbol{E}(t)-2 R C \boldsymbol{P}_{\boldsymbol{b}}(t)\right)}\right)$.

The right side of the inequality in (11) is concave, because it consists of a sum of an affine function $-\boldsymbol{E}(t) /(R C)$ with a geometric mean of non-negative affine functions, which is concave in both $\boldsymbol{E}(t)$ and $\boldsymbol{E}(t)-2 R C \boldsymbol{P}_{\boldsymbol{b}}(t)$ (the nonnegativeness of the latter function comes from (6c)).

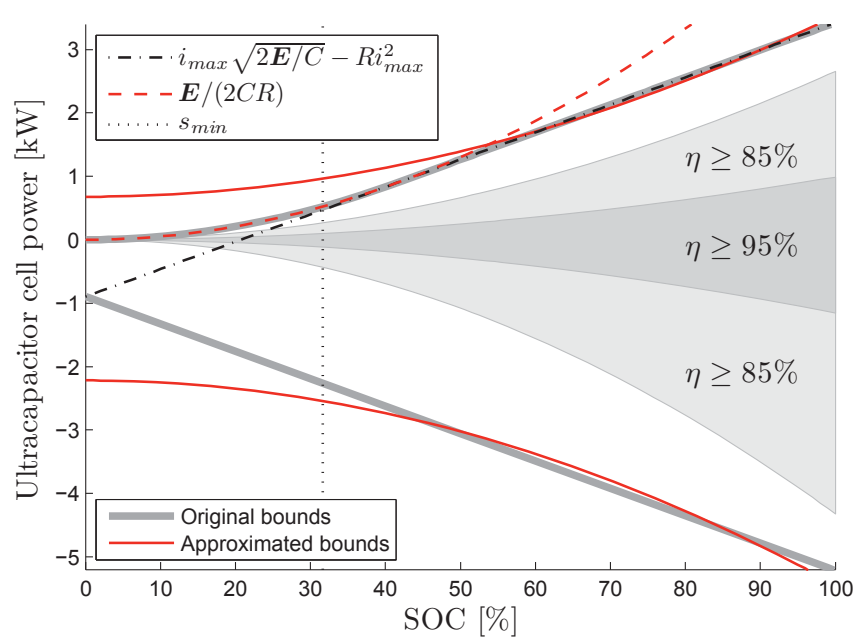

Fig. 5. Bounds on the ultracapacitor cell power (this cell is used later in Section 4). The ultracapacitor is mainly operated within the shaded region, where the efficiency is above $85 \%$.

The integral of (11),

$$
\begin{aligned}
& \boldsymbol{E}(t) \leq \boldsymbol{E}\left(t_{0}\right)+ \\
& \frac{1}{R C} \int_{t_{0}}^{t}\left(\sqrt{\boldsymbol{E}(\tau)\left(\boldsymbol{E}(\tau)-2 R C \boldsymbol{P}_{\boldsymbol{b}}(\tau)\right)}-\boldsymbol{E}(\tau)\right) d \tau
\end{aligned}
$$

of concave functions is concave, giving a constraint that preserves the problem convexity. The relaxation (12) changes the problem, because for a given time interval allows less energy in the buffer than the actual amount corresponding to the cumulative input/output power. However, at the optimum (12) will hold with equality, as otherwise the buffer would throw away energy and the result would not have been optimal.

Accordingly, the constraints (6e), (6f) will change to

$\boldsymbol{E}(t) \in\left[s_{\min }^{2}, 1\right] \frac{C u_{\max }^{2}}{2} \boldsymbol{n}$
$\boldsymbol{E}\left(t_{f}\right)=\boldsymbol{E}\left(t_{0}\right)=s_{0}^{2} \frac{C u_{\max }^{2}}{2} \boldsymbol{n}$.

Bounds on the cell current The bounds on the cell current (6b) can be expressed as bounds on the pack power by applying the current limits $i_{\min }, i_{\max }$ in (7),

$$
\begin{aligned}
\boldsymbol{P}_{\boldsymbol{b}}(t) & \geq \sqrt{\frac{2 \boldsymbol{E}(t) \boldsymbol{n}}{C}} i_{\text {min }}-R i_{\min }^{2} \boldsymbol{n} \\
\boldsymbol{P}_{\boldsymbol{b}}(t) & \leq \begin{cases}\sqrt{\frac{2 \boldsymbol{E}(t) \boldsymbol{n}}{C}} i_{\max }-R i_{\max }^{2} \boldsymbol{n}, & \frac{\boldsymbol{E}(t)}{2 R^{2} C \boldsymbol{n}}>i_{\max }^{2} \\
\boldsymbol{E}(t) /(2 R C), & \text { otherwise }\end{cases}
\end{aligned}
$$

where in (15) the constraint (6c) has also been included. The power bounds are illustrated in Fig. 5, where it can be seen that the upper power bound is associated with $i_{\max }$ down to the point when $\boldsymbol{E}(t)=2 R^{2} C \boldsymbol{n} i_{\max }^{2}$ (power losses become equal to the usable power). With lower energy levels, the maximum power is not associated with $i_{\max }$, but rather to a lower current at which $\partial \boldsymbol{P}_{\boldsymbol{b}}(t) / \partial i(\cdot)=0$ holds (i.e. constraint (6c)).

The constraint (14) is convex since $i_{\min }$ is negative and the geometric mean $\sqrt{\boldsymbol{E}(t) \boldsymbol{n}}$ is concave, giving a convex function on the right side of the inequality. The upper 


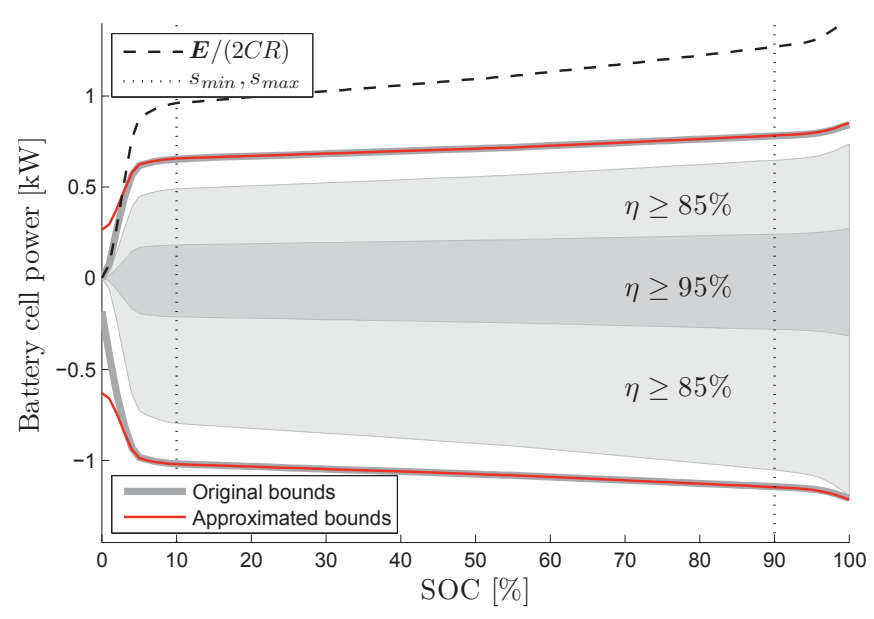

Fig. 6. Bounds on the battery cell power (this cell is used later in Section 4).

bound (15) is concave, but this is not easy to see from the mathematical model. For this reason (15) is expressed with two inequalities

$$
\begin{aligned}
& \boldsymbol{P}_{\boldsymbol{b}}(t) \leq \frac{\boldsymbol{E}(t)}{2 R C} \\
& \boldsymbol{E}(t)-\sqrt{\boldsymbol{E}^{\mathbf{2}}(t)-2 R C \boldsymbol{E}(t) \boldsymbol{P}_{\boldsymbol{b}}(t)} \leq i_{\max } R \sqrt{2 C \boldsymbol{E}(t) \boldsymbol{n}}
\end{aligned}
$$

where (16b) is obtained by multiplying (6b) with $R \sqrt{2 C \boldsymbol{E}(t) \boldsymbol{n}}$. It is now clear that the left side of the inequality in (16b) is convex and the right side is concave, thus preserving the problem convexity. However, both (14) and (16b) are computationally expensive because they are nonlinear (second order cone) constraints (Glineur, 2001). To reduce complexity linear bounds can be used

$\boldsymbol{P}_{\boldsymbol{b}}(t) \geq c_{0} \boldsymbol{E}(t)+c_{1} \boldsymbol{n}$

$\boldsymbol{P}_{\boldsymbol{b}}(t) \leq \min \left\{\frac{\boldsymbol{E}(t)}{2 R C}, c_{2} \boldsymbol{E}(t)+c_{3} \boldsymbol{n}\right\}$

where the pointwise minimum of affine functions in $(17 \mathrm{~b})$ is concave. The coefficients $c_{j}, j=0, \ldots, 3$ in (17) can be found by least squares, or by solving a simple curve fitting convex problem (Boyd and Vandenberghe, 2004), to give good fit within the allowed SOC range. In either case, the approximation error will typically not have significant impact on the optimal result. In HEV applications ultracapacitors are sized by the energy storage requirements because of the relatively low energy density and the high power density (Burke, 2000, 2007). Hence, it is not common that the capacitor will be operated close to the peak power, e.g. close to $\boldsymbol{E}(t) /(2 R C)$, since at that power the efficiency is very low, i.e. close to $50 \%$. A more appropriate power is

$\boldsymbol{P}_{\boldsymbol{b}}(t)= \begin{cases}\eta(1-\eta) \frac{2 \boldsymbol{E}(t)}{R C}, & \boldsymbol{P}_{\boldsymbol{b}}(t) \geq 0 \\ -\frac{1-\eta}{\eta^{2}} \frac{2 \boldsymbol{E}(t)}{R C}, & \text { otherwise }\end{cases}$

at which the efficiency is $\eta$. In Fig. 5 two regions with $\eta \geq 85 \%$ and $\eta \geq 95 \%$ are illustrated.

\subsection{Convex battery model}

The battery open circuit voltage, illustrated in Fig. 4, can be approximated with a linear function $u(\boldsymbol{s}(t))=d_{0} \boldsymbol{s}(t)+d_{1}$

that gives good fit within the allowed SOC range. Similarly as with the ultracapacitor, a variable change is proposed

$\boldsymbol{E}(t)=\frac{C\left(d_{0} \boldsymbol{s}(t)+d_{1}\right)^{2} \boldsymbol{n}}{2}$

where $C=2 Q / \bar{U}$ relates the battery capacity $Q[\mathrm{Ah}]$ with the nominal open circuit voltage $\bar{U}$ (dashed line in Fig. 4) and is used only to show resemblance with the capacitor model. By replacing (19) in (8), and relaxing the obtained constraint (20a) with inequality, the battery constraints can be written in a convex form

$$
\begin{aligned}
& \dot{\boldsymbol{E}}(t) \leq-\frac{d_{0}}{R Q}\left(\boldsymbol{E}(t)-\sqrt{\boldsymbol{E}^{\mathbf{2}}(t)-2 R C \boldsymbol{P}_{\boldsymbol{b}}(t) \boldsymbol{E}(t)}\right) \\
& \boldsymbol{E}(t) \in \frac{C}{2}\left[u^{2}\left(s_{\min }\right), u^{2}\left(s_{\max }\right)\right] \boldsymbol{n} \\
& \boldsymbol{E}\left(t_{f}\right)=\boldsymbol{E}\left(t_{0}\right)=\frac{C}{2} u^{2}\left(s_{0}\right) \boldsymbol{n}
\end{aligned}
$$

similar to the ultracapacitor corresponding equations (11), (13).

The bounds on the cell current can be expressed exactly as in (14), (16). However, similarly as with the ultracapacitor, they can be approximated with linear functions as in (17). The only difference is that the power bound $\boldsymbol{E}(t) /(2 R C)$ cannot be activated due to the battery SOC limit that prevents operation at low charge, see Fig. 6. In this case, the coefficients $c_{j}$ in (17) can be easily computed, if the linear bounds are chosen to pass through the points where the power bounds (14), (15), intersect with the SOC limits $s_{\min }, s_{\max }$.

When using batteries the goodness of the fit is more relevant, because compared to the ultracapacitors, the batteries have lower power density and are likely to be operated close to the power bounds.

\subsection{Approximation of the power losses}

This section shows an alternative method for convexifying the energy buffer by approximating the pack power losses. The approximation is applied to the ultracapacitor model, but the same method can be also used for the battery.

The power losses of the ultracapacitor

$P_{\text {bloss }}(\cdot)=R i^{2}(\cdot) \boldsymbol{n}=\frac{\boldsymbol{n}}{4 R}\left(\boldsymbol{u}(t)-\sqrt{\boldsymbol{u}^{\mathbf{2}}(t)-\frac{4 R \boldsymbol{P}_{\boldsymbol{b}}(t)}{\boldsymbol{n}}}\right)^{2}$

can be approximated with the first three terms of the Taylor series around $\boldsymbol{P}_{\boldsymbol{b}}(t)=0$,

$$
\begin{gathered}
\left.P_{\text {bloss }}(\cdot) \approx P_{\text {bloss }}(\cdot)\right|_{0}+\left.\frac{\partial P_{\text {bloss }}(\cdot)}{\partial \boldsymbol{P}_{\boldsymbol{b}}(t)}\right|_{0} \boldsymbol{P}_{\boldsymbol{b}}(t) \\
+\left.\frac{1}{2} \frac{\partial^{2} P_{\text {bloss }}(\cdot)}{\partial \boldsymbol{P}_{\boldsymbol{b}}^{\mathbf{2}}(t)}\right|_{0} \boldsymbol{P}_{\boldsymbol{b}}^{\mathbf{2}}(t)=\frac{R \boldsymbol{P}_{\boldsymbol{b}}^{\mathbf{2}}(t)}{\boldsymbol{u}^{\mathbf{2}}(t) \boldsymbol{n}} .
\end{gathered}
$$

Using (21) in (7) to express the cell current,

$i(\cdot)=\frac{\boldsymbol{P}_{\boldsymbol{b}}(t)+P_{\text {bloss }}(\cdot)}{\boldsymbol{u}(t) \boldsymbol{n}}$

will give the following state equation

$\dot{\boldsymbol{u}}(t)=-\frac{1}{C}\left(\frac{\boldsymbol{P}_{\boldsymbol{b}}(t)}{\boldsymbol{u}(t) \boldsymbol{n}}+\frac{R \boldsymbol{P}_{\boldsymbol{b}}^{\mathbf{2}}(t)}{\boldsymbol{u}^{\mathbf{3}}(t) \boldsymbol{n}^{\mathbf{2}}}\right)$ 
Table 1. Optimization results.

\begin{tabular}{l|l|l|ll}
\hline \multicolumn{1}{l}{ Energy buffer } & & Capacitor & Battery & \\
\hline Baseline model & $t_{s}$ & 15.3 & 15.1 & $\mathrm{~s}$ \\
(nonlinear power & cost & 3.69 & 4.08 & $€$ \\
bounds, linear & $\boldsymbol{n}$ & 359.1 & 139.1 & \\
voltage-SOC & $f c$ & 20.1 & 21.7 & $1 / 100 \mathrm{~km}$ \\
battery model) & $p_{\text {brk }}$ & 100 & 99.8 & $\%$ \\
\hline \multirow{2}{*}{ Linear power } & $t_{s}$ & 9.8 & 7.4 & $\mathrm{~s}$ \\
bounds & $\boldsymbol{n}$ & 359.1 & 139.1 & \\
& $\delta$ & 0.00 & 0.00 & $\%$ \\
\hline \multirow{2}{*}{ Constant voltage } & $t_{s}$ & 8.4 & 8.3 & $\mathrm{~s}$ \\
& $\boldsymbol{n}$ & 349.3 & 139.4 & \\
\hline \multirow{2}{*}{ Approximated } & $\delta$ & -0.61 & 0.04 & $\%$ \\
power losses & $t_{s}$ & 10.6 & 9.1 & $\mathrm{~s}$ \\
& $\boldsymbol{n}$ & 360.8 & 150.2 & \\
\hline & $\delta$ & 0.07 & 1.17 & $\%$ \\
\hline
\end{tabular}

$t_{s}$ is computation time, cost is total optimal cost,

$f c$ is fuel consumption, $p_{b r k}$ is percent of recuperated braking energy.

which after the variable change (10) and the relaxation with inequality, becomes

$$
\dot{\boldsymbol{E}}(t) \leq-\left(\boldsymbol{P}_{\boldsymbol{b}}(t)+\frac{R C}{2} \frac{\boldsymbol{P}_{\boldsymbol{b}}^{\mathbf{2}}(t)}{\boldsymbol{E}(t)}\right) .
$$

The upper bound on the cell current can be written either as in (16), or the approximation (22) could be used to replace $(16 \mathrm{~b})$ with

$$
\boldsymbol{P}_{\boldsymbol{b}}(t)+\frac{R C}{2} \frac{\boldsymbol{P}_{\boldsymbol{b}}^{\mathbf{2}}(t)}{\boldsymbol{E}(t)} \leq i_{\max } \sqrt{\frac{2 \boldsymbol{E}(t) \boldsymbol{n}}{C}} .
$$

The rest of the constraints are as in (13), (14). To avoid division by zero, $\boldsymbol{E}(t)$ must be strictly positive, which is a sufficient condition for convexity of the quadratic-overlinear function $\boldsymbol{P}_{\boldsymbol{b}}^{\mathbf{2}}(t) / \boldsymbol{E}(t)$.

\section{EXAMPLE OF OPTIMAL BUFFER SIZING}

This section gives an example of buffer sizing and optimal control of an HEV city bus. The energy buffer can be built either from battery cells, or ultracapacitor cells. The losses of the power electronics are assumed, for simplicity, identical for the two energy buffers.

\subsection{Problem setup}

The bus is equipped with a $220 \mathrm{~kW}$ EM and a $180 \mathrm{~kW}$ EGU as in Fig. 2. The battery cells considered for the energy buffer have capacity of $45 \mathrm{Ah}$, current limits of $\pm 250 \mathrm{~A}$ and cost $500 € / \mathrm{kWh}$, while the ultracapacitor cells have capacity of $2000 \mathrm{~F}$, current limits of $\pm 1600 \mathrm{~A}$ and cost $10000 € / \mathrm{kWh}$. The remaining details of both cells are available online ${ }^{1}$. The operation is charge sustaining, where it is required to start and end at half of the total energy content (about 50\% SOC for the battery and $71 \%$ SOC for the ultracapacitor). The bus line is a standard emission test cycle ${ }^{2}$.

\footnotetext{
1 The battery cell is manufactured by Saft (http://www saftbatteries.com) in lithium-ion technology and can be found under the name VL 45E. The ultracapacitor cell is manufactured by Maxwell (http://www.maxwell.com/) and can be found in the K2 series under the name BCAP2000 P270 (March 2012).

2 The test cycle, City Suburban Cycle, can be found on http://www.dieselnet.com/standards/cycles (March 2012).
}

The convex problem is written in time discrete form, which for the HEV with an ultracapacitor and no approximations, can be summarized as

minimize

$w_{f} h \sum_{0}^{N-1} P_{f}\left(\boldsymbol{P}_{\boldsymbol{g}}(k)\right)+w_{b} \boldsymbol{n}$

$\forall k \in[0, N-1]$, subject to

$\boldsymbol{P}_{\boldsymbol{b}}(k) \geq \sqrt{\frac{2 \boldsymbol{E}(k) \boldsymbol{n}}{C}} i_{\text {min }}-R i_{\text {min }}^{2} \boldsymbol{n}$

$\boldsymbol{P}_{\boldsymbol{b}}(k) \leq \frac{\boldsymbol{E}(k)}{2 R C}$

$\boldsymbol{E}(k)-\sqrt{\boldsymbol{E}^{\mathbf{2}}(k)-2 R C \boldsymbol{E}(k) \boldsymbol{P}_{\boldsymbol{b}}(k)} \leq i_{\max } R \sqrt{2 C \boldsymbol{E}(k) \boldsymbol{n}}$

$\boldsymbol{E}(k+1) \leq \boldsymbol{E}(k)\left(1-\frac{h}{R C}\right)+\frac{h \sqrt{\boldsymbol{E}^{2}(k)-2 R C \boldsymbol{P}_{\boldsymbol{b}}(k) \boldsymbol{E}(k)}}{R C}$

$\boldsymbol{E}(k) \in\left[s_{\min }^{2}, 1\right] \frac{C u_{\max }^{2}}{2} \boldsymbol{n}$

$\boldsymbol{E}(N)=\boldsymbol{E}(0)=s_{0}^{2} \frac{C u_{\max }^{2}}{2} \boldsymbol{n}$

$\boldsymbol{T}(k) \geq \max \left\{T_{\min }(\omega(k)), T_{n b}(k)+\boldsymbol{n} T_{b}(k)\right\}$

$\boldsymbol{T}(k) \omega(t)+P_{\text {EMloss }}(\boldsymbol{T}(k), k) \leq \boldsymbol{P}_{\boldsymbol{b}}(k)+\boldsymbol{P}_{\boldsymbol{g}}(k)-P_{a}$

$\boldsymbol{P}_{\boldsymbol{g}}(k) \in\left[0, P_{\text {gmax }} e(k)\right]$

$\boldsymbol{n} \geq 0$.

The derivative (11) has been replaced with a first order forward Euler discretization with a sampling time of $h=$ 1 s, giving $N=1700$ time samples for the chosen bus line.

The optimization variables are scaled and a parser is used, CVX (Grant and Boyd, 2010), to translate the problem in the form required by the solver SeDuMi (Labit et al., 2002).

\subsection{Optimization results}

The optimal results of battery and ultracapacitor sizing are given in Table 1. The ultracapacitor model without approximations and the battery model with linear voltageSOC relationship are being denoted as baseline models. Then, the optimal cost of the baseline models is used to validate the approximation error

$\delta=\frac{\text { cost approximated }- \text { cost baseline }}{\text { cost baseline }} \times 100$

for three different approximated battery and ultracapacitor models. The first approximated models use linear power bounds, as explained in Section 3.2 and 3.3. The second approximated models use constant open circuit voltage, where the battery voltage is the nominal voltage and the ultracapacitor voltage is chosen $u_{\max } / \sqrt{2}$ to give the same energy content as the original model. Details on the convex modeling of a constant voltage buffer have been described by Murgovski et al. (2012). The third approximated models use linear power bounds and approximated power losses as explained in Section 3.4.

The optimal operating points of the energy buffer are given in Fig. 7, and the optimal SOC trajectories along the bus line are given in Fig. 8. The results show that the ultracapacitor is sized by the energy requirements, since it is operated close to the SOC limits, but far from the power limits. On the contrary, the battery is sized by the power 

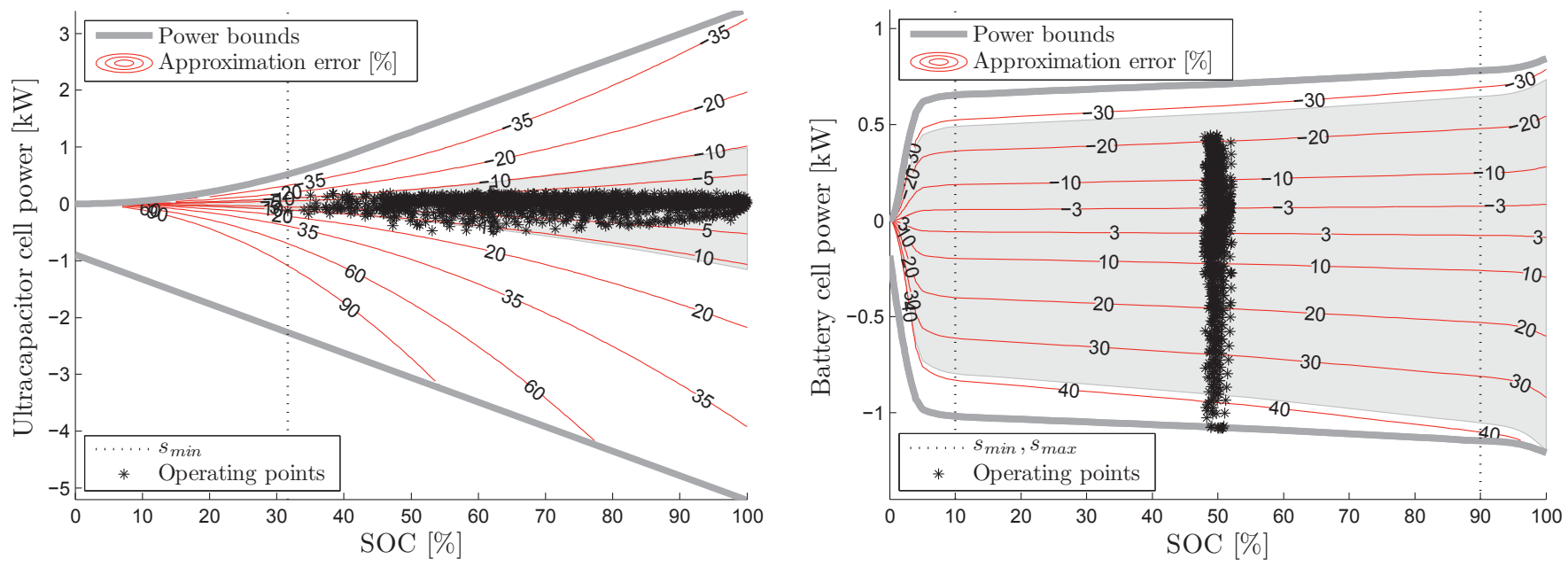

Fig. 7. Optimal operating points of the baseline energy buffer models, one point for each time instant $k$. The shaded regions show efficiency greater than $95 \%$ for the ultracapacitor and $85 \%$ for the battery. The thin contour lines show relative error in power losses when the losses are approximated with (21).

requirements as the SOC is far from the limits, but the power is close to the charging limit. This indicates that both buffers are sized to recuperate most of the free of charge braking energy, that is, the ultracapacitor recovers all the braking energy, while the battery recovers $99.8 \%$.

The capacitor is operated with very high efficiency, mainly above $95 \%$, at any point along the bus line. During discharge, the battery is also operated with high efficiency, above $88 \%$, but the efficiency may drop below $83 \%$ during brake regeneration. This is the reason that the optimization error

error $=\frac{\text { losses approximated }- \text { losses baseline }}{\text { losses baseline }} \times 100$

from approximation of the power losses is lower for the ultracapacitor, as it is directly related to the buffer efficiency. Moreover, Fig. 7 shows that many operating points of the battery will have high approximation error, although the cumulative error given in Table 1 is small, but not negligible $(1.17 \%)$. This is because the approximation underestimates the power losses during discharging, but it overestimates them during charging. As a result, the approximation resulted in $8 \%$ more cells than the baseline, although there is no visible change in the optimal SOC trajectory, as can be seen in the bottom plot of Fig. 8 .

The approximation with linear power bounds gave zero optimization error and managed to speed up the computation time ${ }^{3}$ by up to $50 \%$.

The results in Table 1 also show that the model with approximated constant voltage gave small error, and not only for the battery, but also for the ultracapacitor. The optimal number of ultracapacitor cells, in this case, decreased by $2.7 \%$ and there is small deviation in the optimal SOC trajectory (Fig. 8).

\footnotetext{
3 All optimizations are carried on a standard $\mathrm{PC}$ with $2.67 \mathrm{GHz}$ dual
} core $\mathrm{CPU}$ and $4 \mathrm{~GB}$ RAM.
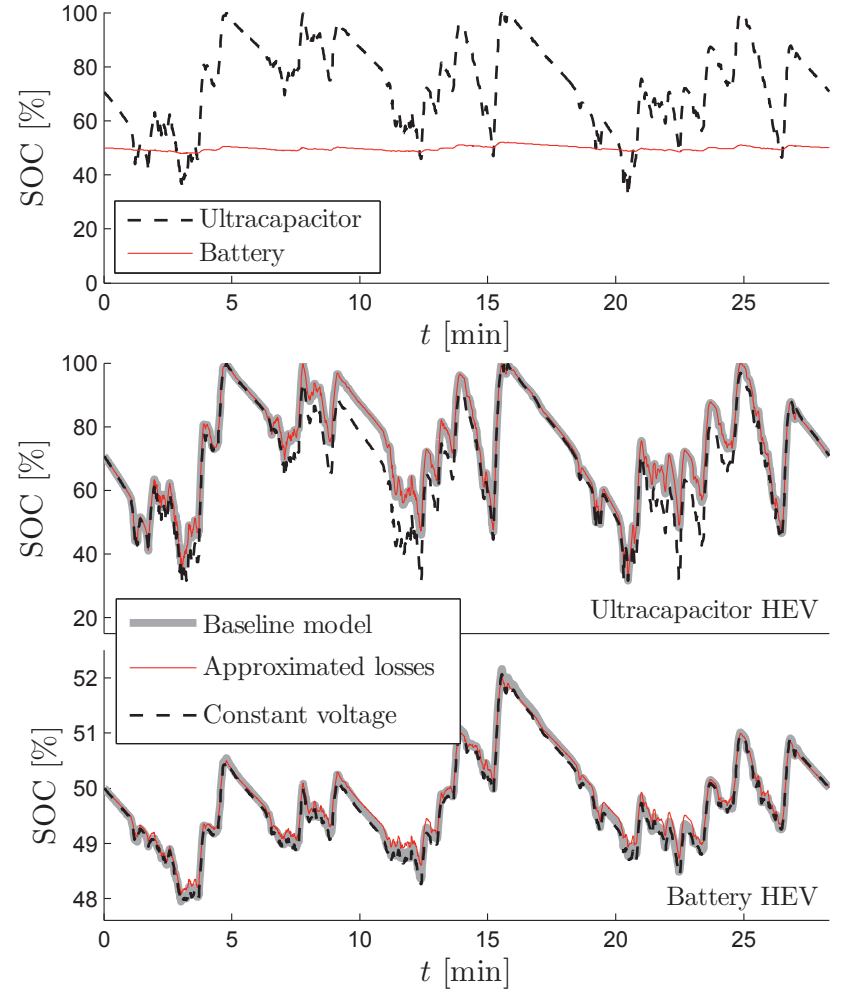

Fig. 8. The top plot shows optimal SOC trajectories for the baseline energy buffer models. The two plots below show, in addition, the optimal SOC trajectories for the models with approximated power losses and the models with approximated constant voltage.

\section{CONCLUSION}

This paper described modeling steps that enable convex optimization for applications with optimal control and simultaneous dimensioning of capacitors and batteries. The paper also showed that by approximating the buffer power bounds with linear functions, the computation time may decrease by up to $50 \%$, with zero optimization 
Table A.1. Parameter values.

\begin{tabular}{ll} 
Vehicle frontal area & $A=7.54 \mathrm{~m}^{2}$ \\
Aerodynamic drag coefficient & $c_{d}=0.7$ \\
Rolling resistance coefficient & $c_{r}=0.007$ \\
Wheel radius & $r=0.509 \mathrm{~m}$ \\
Final gear & $\gamma=4.7$ \\
Vehicle mass without the battery & $m=14.5 \mathrm{t}$ \\
EM inertia & $I_{E M}=2.3 \mathrm{kgm}^{2}$ \\
Inertia of final gear and wheels & $I=41.8 \mathrm{kgm}^{2}$ \\
Power used by auxiliaries & $P_{a}=7 \mathrm{~kW}$ \\
Fuel price & $w_{f}=0.15 € / \mathrm{kWh}$ \\
Average travel distance in one year & $d=80000 \mathrm{~km}$ \\
\hline
\end{tabular}

error. Moreover, modeling steps have been shown for an alternative convex model with approximated power losses of the energy buffer. A result of this study is that even a constant voltage approximation may be a viable approach for the ultracapacitor sizing problem in an HEV city bus.

Besides simultaneous dimensioning and control of HEV energy buffers, the convex models may also allow time efficient optimization of series or parallel powertrain models with several state variables, e.g. thermal states of the components. Extending the convex modeling method on thermal buffer models, and applying the use of convex buffer models in other control applications will be considered in future studies.

\section{Appendix A. OPTIMIZATION DATA}

Denoting by $v(t)$ and $\alpha(t)$ the velocity and slope of the bus line, the angular velocity $\omega(t)$ and torque demanded on the shaft between the EM and the differential, $T_{n b}(t)+\boldsymbol{n} T_{b}(t)$, can be computed as

$$
\begin{aligned}
\omega(t) & =\frac{\gamma}{r} v(t) \\
T_{n b}(t) & =\left(I_{E M}+\frac{I}{\gamma^{2}}+m \frac{r^{2}}{\gamma^{2}}\right) \dot{\omega}(t)+\frac{\rho A c_{d} r^{3}}{2 \gamma^{3}} \omega^{2}(t) \\
& +\frac{g r}{\gamma} m\left(c_{r} \cos \alpha(t)+\sin \alpha(t)\right) \\
T_{b}(t) & =\frac{m_{b c} r}{\gamma}\left(\frac{r}{\gamma} \dot{\omega}(t)+g c_{r} \cos \alpha(t)+g \sin \alpha(t)\right)
\end{aligned}
$$

where $g$ is gravitational acceleration, $\rho$ is air density, and the rest of the parameters are described in Table A.1. The model neglects the inertial effects of the EGU.

The battery cell has mass of $1.22 \mathrm{~kg}$ and inner resistance of $2.9 \mathrm{~m} \Omega$. The ultracapacitor cell has mass of $0.36 \mathrm{~kg}$, inner resistance of $0.35 \mathrm{~m} \Omega$ and maximum voltage of $2.7 \mathrm{~V}$. The additional mass for packaging and circuitry is assumed to be $14 \%$ of the buffer mass.

The payment for the energy buffer is equally divided in $y=5$ years with $p=5 \%$ yearly interest rate. By denoting with $c_{b}$ the buffer price in curency $/ \mathrm{kWh}$, the equivalent cell cost related to the driven bus line is obtained by multiplying the length of the bus line with the cell price per kilometer, given the average travel distance in one year d. This yields

$w_{b}=c_{b} E_{s} m_{b c}\left(1+p \frac{y+1}{2}\right) \frac{\int_{t_{0}}^{t_{f}} v(t) d t}{y d}$

where $E_{s}[\mathrm{kWh} / \mathrm{kg}]$ is specific energy of the entire energy content of the cell.

\section{REFERENCES}

C. D. Anderson and J. Anderson. Electric and hybrid cars, a history. McFarland \& Company, Inc., 2 edition, 2010.

R. Bellman. Dynamic Programming. Princeton Univ Pr, New Jersey, June 1957.

S. Boyd and L. Vandenberghe. Convex Optimization. Cambridge University Press, 2004.

A. F. Burke. Ultracapacitors: why, how, and where is the technology. Journal of Power Sources, 91:37-50, November 2000.

A. F. Burke. Batteries and ultracapacitors for electric, hybrid, and fuel cell vehicles. Proceedings of the IEEE, 95(4):806-820, April 2007.

F. Glineur. Topics In Convex Optimization: Interior-Point Methods, Conic Duality and Approximations. PhD thesis, Mathematics and Operational Research, University of Mons, Belgium, 2001.

M. Grant and S. Boyd. CVX: Matlab software for disciplined convex programming, version 1.21. http://cvxr.com/cvx, May 2010.

L. Guzzella and A. Sciarretta. Vehicle propulsion systems, introduction to modeling and optimization. Springer, Berlin, Heidelberg, 2nd edition, 2007.

L. Johannesson, S. Pettersson, and B. Egardt. Predictive energy management of a 4QT series-parallel hybrid electric bus. Control Engineering Practice, 17(12):14401453, December 2009.

M. Kim and H. Peng. Power management and design optimization of fuel cell/battery hybrid vehicles. Journal of Power Sources, 165(2):819-832, 2007.

Y. Labit, D. Peaucelle, and D. Henrion. SeDuMi interface 1.02: a tool for solving LMI problems with SeDuMi. IEEE International Symposium on Computer Aided Control System Design Proceedings, pages 272277, September 2002.

T. C. Moore. HEV control strategy: Implications of performance criteria, system configuration and design, and component selection. In Proceedings of the American Control Conference, Albuquerque, New Mexico, June 1997.

S. J. Moura, D. S. Callaway, H. K. Fathy, and J. L. Stein. Tradeoffs between battery energy capacity and stochastic optimal power management in plug-in hybrid electric vehicles. Journal of Power Sources, 195(9):29792988, 2010.

N. Murgovski, J. Sjöberg, and J. Fredriksson. A methodology and a tool for evaluating hybrid electric powertrain configurations. Int. J. Electric and Hybrid Vehicles, 3 (3):219-245, 2011.

N. Murgovski, L. Johannesson, J. Sjöberg, and B. Egardt. Component sizing of a plug-in hybrid electric powertrain via convex optimization. Journal of Mechatronics, 22 (1):106-120, 2012.

O. Sundström, L. Guzzella, and P. Soltic. Torque-assist hybrid electric powertrain sizing: From optimal control towards a sizing law. IEEE Transactions on Control Systems Technology, 18(4):837-849, July 2010.

U. Zoelch and D. Schroeder. Dynamic optimization method for design and rating of the components of a hybrid vehicle. International Journal of Vehicle Design, 19(1):1-13, 1998. 